\title{
Positive solutions for some indefinite nonlinear eigenvalue elliptic problems with Robin boundary conditions
}

\author{
H. Ramos Quoirin ${ }^{1}$, ANd A. SuÁrez ${ }^{2}$ \\ 1. Universidad de Santiago de Chile, \\ Casilla 307, Correo 2, Santiago, Chile \\ 2. Dpto. de Ecuaciones Diferenciales y Análisis Numérico \\ Fac. de Matemáticas, Univ. de Sevilla \\ C/. Tarfia s/n, 41012 - Sevilla, Spain, \\ E-mail addresses: humberto.ramos@usach.cl, suarez@us.es
}

\begin{abstract}
We consider a nonlinear eigenvalue problem with indefinite weight under Robin boundary condition. We prove the existence and multiplicity of positive solutions. To this end, we carry out a detailed study of some linear eigenvalues problems and we use mainly bifurcation and sub-supersolution methods.
\end{abstract}

Key Words. Elliptic equations, Indefinite weight, Robin boundary conditions.

\section{Introduction and main results}

Let $\Omega \subset \mathbb{R}^{N}, N \geq 2$, be a bounded domain with a $C^{2, \gamma}$ boundary, $0<\gamma<1$. We are interested in the study of positive solutions for the problem

$$
\left\{\begin{aligned}
-\Delta u & =\lambda m(x)\left(u-u^{2}\right) & & \text { in } \Omega, \\
\frac{\partial u}{\partial \nu} & =\alpha u & & \text { on } \partial \Omega,
\end{aligned}\right.
$$

where $\lambda, \alpha \in \mathbb{R}, m \in C^{1}(\bar{\Omega})$ changes sign and $\nu$ is the outward unit normal to $\partial \Omega$.

Throughout this article we assume that

$$
\int_{\Omega} m<0
$$

since the case $\int_{\Omega} m>0$ reduces to (1.1) changing $\lambda$ by $-\lambda$. The case $\int_{\Omega} m=0$ is singular and will be treated elsewhere.

We shall treat $(P)$ by a bifurcation approach, so we shall consider the linear eigenvalue problem

$$
\left\{\begin{aligned}
-\Delta u & =\lambda m(x) u & & \text { in } \Omega, \\
\frac{\partial u}{\partial \nu} & =\alpha u & & \text { on } \partial \Omega .
\end{aligned}\right.
$$




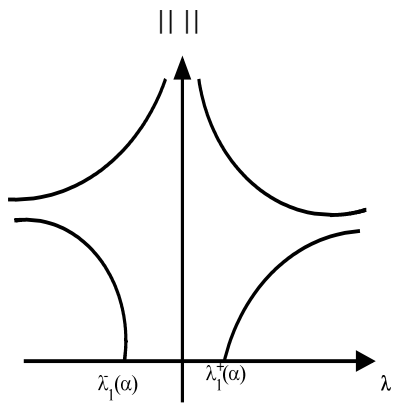

a)

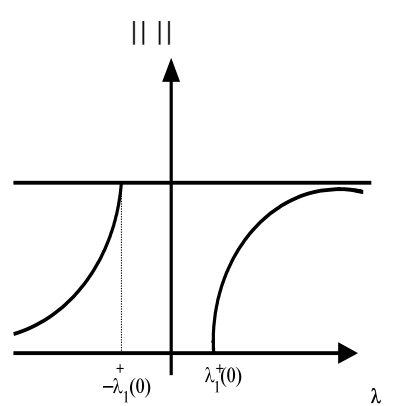

b)

Figure 1: Bifurcation diagrams of $(P)$ : Case a) $\alpha<0$ and Dirichlet boundary conditions. Case b) $\alpha=0$.

It is shown in [1] that there exists $\alpha_{0}^{*}>0$ such that, for $\alpha<\alpha_{0}^{*}$, (E) possesses two principal eigenvalues, denoted by $\lambda_{1}^{-}(\alpha)$ and $\lambda_{1}^{+}(\alpha)$. In the homogeneous Dirichlet boundary conditions case, we denote them by $\lambda_{1}^{ \pm}(D)$. In Section 2 we recall the results from [1] and complement them providing an expression for $\alpha_{0}^{*}$.

$(P)$ has already been studied in different cases. For the cases $\alpha<0$ [7] and Dirichlet boundary conditions $[2,11]$, it has been proved that $(P)$ has a positive solution for all $\lambda \neq 0$ and, under further conditions for a priori bounds, at least two positive solutions for $\lambda \in\left(-\infty, \lambda_{1}^{-}(\alpha)\right) \cup\left(\lambda_{1}^{+}(\alpha),+\infty\right)$ and $\lambda \in\left(-\infty, \lambda_{1}^{-}(D)\right) \cup\left(\lambda_{1}^{+}(D),+\infty\right)$, respectively. See Figure 1 (a) for the bifurcation diagram in these cases.

The case $\alpha=0$, which has been analyzed in [6] (see also [14, 17]), is singular in the following sense: the trivial solutions $u \equiv 0$ and $u \equiv 1$ exist for all $\lambda \in \mathbb{R}$, and for $\lambda=0$ the positive constants are solutions. Moreover, for $\lambda \in\left(-\infty,-\lambda_{1}^{+}(0)\right) \cup\left(\lambda_{1}^{+}(0),+\infty\right)$ there exists a stable solution $u<1$, which is the only positive solution of $(P)$ less than one, see Figure 1 (b). Recall that in this case $\lambda_{1}^{-}(0)=0$.

Finally, the case $\alpha>0$ and small was studied in [7]. Assuming $2<(N+2) /(N-2)$ and using variational methods, the authors proved that if $0<\alpha<\alpha_{0}^{*}$ and $\lambda \in\left(\lambda_{1}^{-}(\alpha), \lambda_{1}^{+}(\alpha)\right)$ then $(P)$ possesses at least a positive solution.

In this article, we adopt a different viewpoint, namely, we consider $\lambda$ fixed and look at $\alpha$ as a bifurcation parameter. Consequently, we improve some results of [6] for $\alpha=0$, and complement the study of $(P)$ when $\alpha>0$.

We shall assume that

$$
M_{ \pm}:=\left\{x \in \Omega: m^{ \pm}>0\right\}
$$

are open and regular sets; here $m^{ \pm}$denote the positive and negative part of $m$ respectively. We shall also assume that $m^{ \pm}(x) \approx\left[\operatorname{dist}\left(x, \partial M_{ \pm}\right)\right]^{\gamma^{ \pm}}$for $x$ close to $\partial M_{ \pm}$and some $\gamma_{ \pm} \geq 0$. Let

$$
M_{0}:=\Omega \backslash\left(\overline{M_{+}} \cup \overline{M_{-}}\right) .
$$

We assume the following conditions on $M_{ \pm}$and $M_{0}$ : 

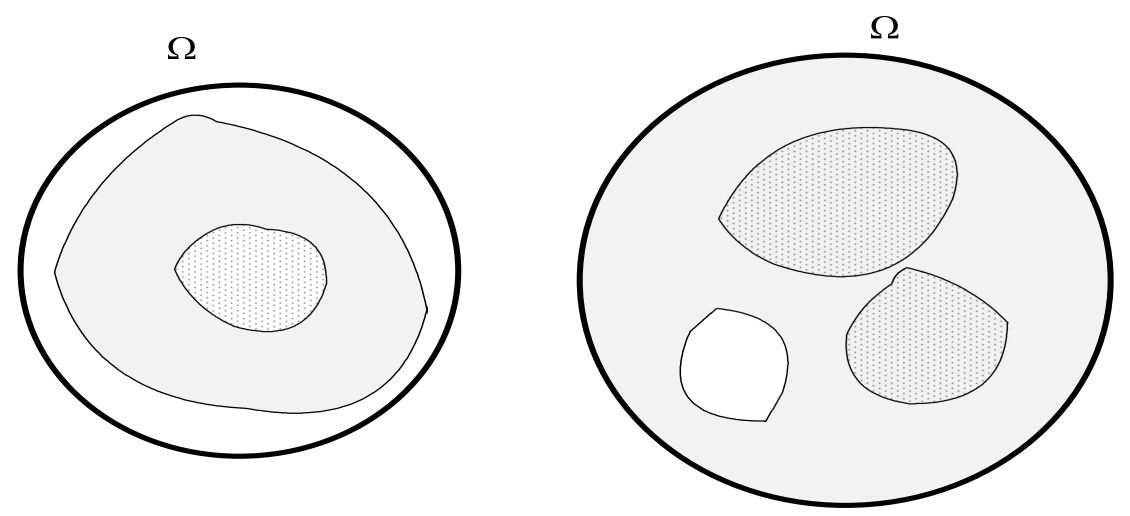

Figure 2: Two examples of admissible domains. The white, shady and lined sets represent $M_{0}, M_{+}$and $M_{-}$, respectively.

$\left(H_{M_{0}}\right) M_{0}$ is a proper subdomain of $\Omega$, i.e. $\operatorname{dist}\left(\partial \Omega, \partial M_{0} \cap \Omega\right)>0$.

$\left(H_{M_{ \pm}}\right) \partial M_{ \pm}=\Gamma_{1}^{ \pm} \cup \Gamma_{2}^{ \pm}$, with $\Gamma_{1}^{ \pm}=\partial \Omega \cap \partial M_{ \pm}$and $\Gamma_{2}^{ \pm} \subset \Omega$.

In fact, $\left(H_{M_{ \pm}}\right)$is assumed to avoid regularity issues, see [12]. In Figure 2 we have represented two different admissible domains.

Our first result is related to a priori bounds for positive solutions of $(P)$. We show that if

$$
2<\min \left\{\frac{N+2}{N-2}, \frac{N+1+\gamma^{ \pm}}{N-1}\right\}
$$

then, there exist a priori bounds for positive solutions of $(P)$ whenever $\alpha$ varies in compact sets of $\mathbb{R}$.

In order to show our main results, we need to introduce some further notation. We denote by $\lambda_{1}(-\Delta-\lambda m, N)$ and $\lambda_{1}(-\Delta-\lambda m, D)$ the principal eigenvalues of the problem

$$
-\Delta \varphi-\lambda m(x) \varphi=\sigma \varphi \text { in } \Omega
$$

under homogenous Neumann and Dirichlet boundary conditions, respectively. In Section 2 , we show that given $\lambda \in \mathbb{R}$, there exists a principal eigenvalue of $(E)$ with respect to $\alpha$, denoted by $\alpha_{1}(\lambda)$, if and only if $\lambda_{1}(-\Delta-\lambda m, D)>0$. Furthermore, $\operatorname{sign}\left(\alpha_{1}(\lambda)\right)=$ $\operatorname{sign}\left(\lambda_{1}(-\Delta-\lambda m, N)\right)$.

Note that if $\lambda=0$ then $(P)$ has no positive solutions unless if $\alpha=0$, in which case, all the positive constants are solutions. So we assume that $\lambda \neq 0$ along this article.

We state now our main result (see Figure 3):

Theorem 1.1. Assume (1.1) and (1.3).

1. Assume $\lambda_{1}(-\Delta-\lambda m, D)>0$. Then there exists $\alpha_{*} \geq \alpha_{1}(\lambda)$ such that $(P)$ has a positive solution if $\alpha<\alpha_{*}$ and no positive solution for $\alpha>\alpha_{*}$. Moreover, there exists $\alpha_{* *} \in\left(\alpha_{1}(\lambda), \alpha_{*}\right]$ such that $(P)$ has at least two positive solutions for $\alpha \in$ $\left(\alpha_{1}(\lambda), \alpha_{* *}\right)$. In addition: 
(a) If $\lambda_{1}(-\Delta-\lambda m, N)>0$ then $0<\alpha_{1}(\lambda) \leq \alpha_{* *}$.

(b) If $\lambda_{1}(-\Delta-\lambda m, N)=0$ then $0=\alpha_{1}(\lambda)<\alpha_{* *}$.

(c) If $\lambda_{1}(-\Delta-\lambda m, N)<0$ and $\lambda \neq-\lambda_{1}^{+}(0)$ then $\alpha_{1}(\lambda)<0<\alpha_{* *}$.

(d) If $\lambda=-\lambda_{1}^{+}(0)$ then $\alpha_{1}(\lambda)<0 \leq \alpha_{* *}$.

2. Assume $\lambda_{1}(-\Delta-\lambda m, D) \leq 0$. Then there exist $\alpha_{*}>0$ such that $(P)$ has a positive solution if and only if $\alpha \leq \alpha_{*}$. Moreover, there exists $\alpha_{* *} \in\left(0, \alpha_{*}\right]$ such that $(P)$ has at least two positive solutions for $\alpha<\alpha_{* *}$.

As a consequence, we obtain (see Figure 4 (a)):

Theorem 1.2. Assume (1.1) and $\alpha=0$.

1. For all $\lambda \in \mathbb{R}, u \equiv 1$ is a positive solution of $(P)$, which is stable for $\lambda \in\left(-\lambda_{1}^{+}(0), 0\right)$.

2. $(P)$ has a second (and stable) positive solution for $\lambda>\lambda_{1}^{+}(0)$ and $\lambda<-\lambda_{1}^{+}(0)$.

3. Assume (1.3). (P) has a second positive solution for $-\lambda_{1}^{+}(0)<\lambda<0$.

In the case $\alpha>0$, we get:

Theorem 1.3. Assume (1.1), (1.3) and $\alpha>0$.

1. There exists $\alpha_{0}>0$, such that $(P)$ has no positive solution for $\alpha \geq \alpha_{0}$.

2. Let $\lambda<0$ and $\lambda \neq-\lambda_{1}^{+}(0)$. Then there exists $\alpha^{*}(\lambda)$ such that $(P)$ has at least two positive solutions for $\alpha<\alpha^{*}(\lambda)$.

3. Let $\lambda \in\left(\lambda_{1}^{-}(\alpha), \lambda_{1}^{+}(\alpha)\right)$ and $0<\alpha<\alpha_{0}^{*}$. Then $(P)$ has at least a positive solution.

4. Let $\lambda \geq \lambda_{1}^{+}(0)$. Then $(P)$ has at least two positive solutions for $\alpha$ sufficiently small.

We stress that we do not know what the bifurcation diagram looks like in the case $\lambda \in\left[\lambda_{1}^{+}(\alpha), \lambda_{1}^{+}(0)\right)$. However, since $\lambda_{1}^{+}(\alpha) \rightarrow \lambda_{1}^{+}(0)$ as $\alpha \rightarrow 0$, we have represented in Figure 4 (b) the suggested bifurcation diagram in the case $\alpha>0$ and small.

The outline of this article is as follows: in Section 2 we study in detail the eigenvalue problems related to $(P)$. In Section 3 we consider $(P)$ with $\alpha$ as the bifurcation parameter. Finally, Section 4 is devoted to prove our main results.

\section{Eigenvalue problems}

Given $m \in L^{\infty}(\Omega)$ and $h \in C^{1}(\partial \Omega)$, we denote by $\lambda_{1}(-\Delta+m, N+h)$ the principal eigenvalue (the notation $N$ refers to the Neumann boundary condition) of the problem

$$
\begin{cases}-\Delta u+m(x) u=\lambda u & \text { in } \Omega, \\ \frac{\partial u}{\partial \nu}+h(x) u=0 & \text { on } \partial \Omega .\end{cases}
$$

Let us summarize the main properties of $\lambda_{1}(-\Delta+m, N+h)$. For a proof, we refer to [8]:

Lemma 2.1. $\lambda_{1}(-\Delta+m, N+h)$ is a simple eigenvalue, and any eigenfunction $\varphi$ associated to $\lambda_{1}(-\Delta+m, N+h)$ satisfies $\varphi \in C^{1, \gamma}(\bar{\Omega}) \cap H^{2}(\Omega), \gamma \in(0,1)$. In addition: 


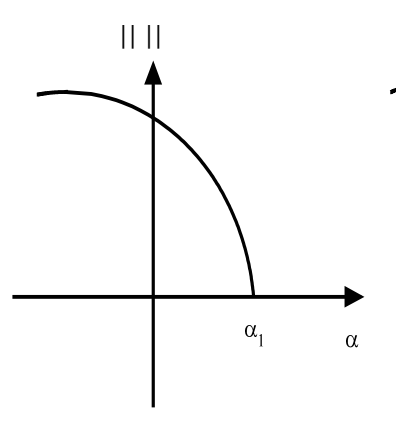

a)

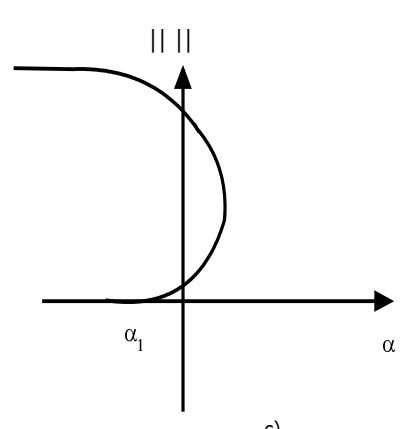

c)

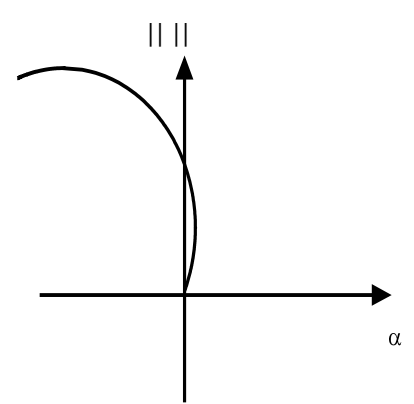

b)

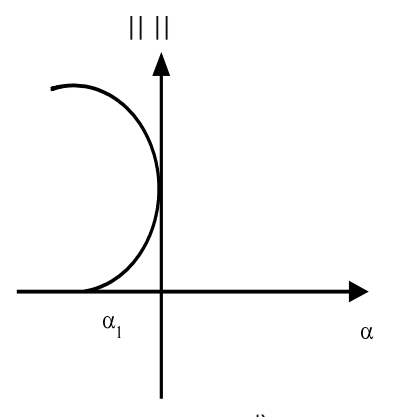

d)

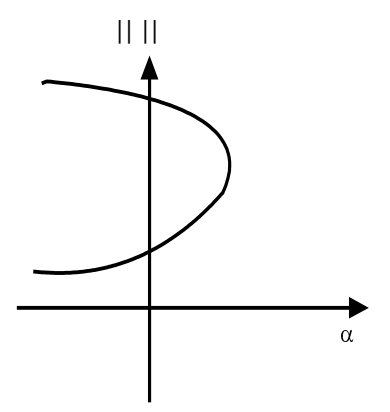

e)

Figure 3: Bifurcation diagrams of $(P)$ : Case a) $\lambda_{1}(-\Delta-\lambda m, N)>0$. Case b) $\lambda_{1}(-\Delta-$ $\lambda m, N)=0$. Case c) $\lambda_{1}(-\Delta-\lambda m, N)<0<\lambda_{1}(-\Delta-\lambda m, D)$ and $\lambda \neq-\lambda_{1}^{+}(0)$. Case d) $\lambda=-\lambda_{1}^{+}(0)$. Case e) $\lambda_{1}(-\Delta-\lambda m, D) \leq 0$. 


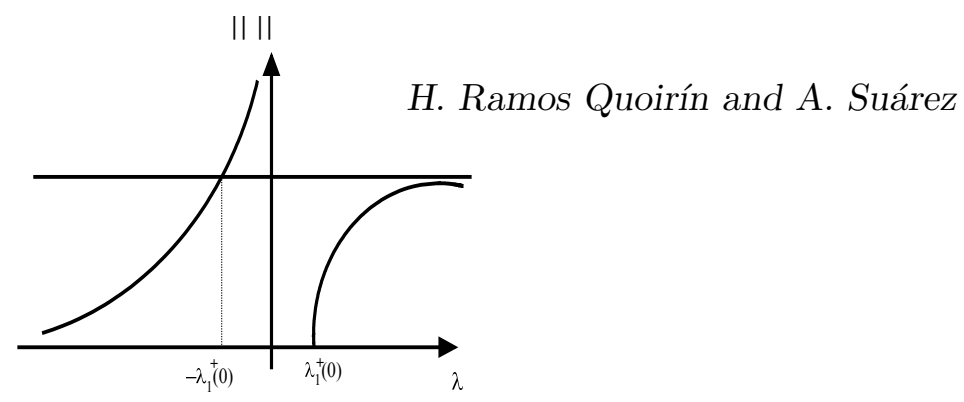

a)

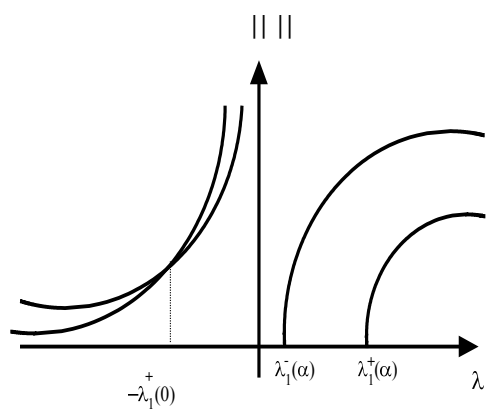

b)

Figure 4: Bifurcation diagrams of $(P)$ : Case a) $\alpha=0$. Case b) $\alpha>0$ and small.

1. $\lambda_{1}(-\Delta+m, N+h)$ is separately increasing with respect to $m$ and $h$.

2. $\lambda_{1}(-\Delta+m, N+h)<\lambda_{1}(-\Delta+m, D)$ where $\lambda_{1}(-\Delta+m, D)$ stands for the principal eigenvalue of $-\Delta+m$ with homogeneous Dirichlet boundary conditions.

3. Assume that $G \subset \Omega$ is a proper regular subdomain of $\Omega$, that is,

$$
\operatorname{dist}(\partial \Omega, \partial G \cap \Omega)>0
$$

and denote by $\lambda_{1}^{G}(-\Delta+m, N+h, D)$ the principal eigenvalue of

$$
\begin{cases}-\Delta u+m(x) u=\lambda u & \text { in } G, \\ \frac{\partial u}{\partial \nu}+h(x) u=0 & \text { on } \partial G \cap \partial \Omega, \\ u=0 & \text { on } \partial G \cap \Omega .\end{cases}
$$

Then

$$
\lambda_{1}(-\Delta+m, N+h)<\lambda_{1}^{G}(-\Delta+m, N+h, D) .
$$

4. There holds

$$
\begin{aligned}
& \lim _{K \rightarrow-\infty} \lambda_{1}(-\Delta+m, N+K)=-\infty, \\
& \lim _{K \rightarrow+\infty} \lambda_{1}(-\Delta+m, N+K)=\lambda_{1}(-\Delta+m, D) .
\end{aligned}
$$


Indefinite elliptic problem and Robin boundary conditions

Given $\lambda, \alpha \in \mathbb{R}$, we set

$$
\mu(\lambda, \alpha):=\lambda_{1}(-\Delta-\lambda m, N-\alpha)
$$

and

$$
I_{\lambda, \alpha}(u)=\int_{\Omega}\left(|\nabla u|^{2}-\lambda m(x) u^{2}\right)-\alpha \int_{\partial \Omega} u^{2} \quad \text { for } \quad u \in H^{1}(\Omega) .
$$

Recall that

$$
\mu(\lambda, \alpha)=\min \left\{I_{\lambda, \alpha}(u) ; u \in H^{1}(\Omega), \int_{\Omega} u^{2}=1\right\} .
$$

This map has the following properties, which follow from Lemma 2.1 and [1, Lemma 2]:

\section{Lemma 2.2.}

1. The map $\alpha \mapsto \mu(\lambda, \alpha)$ is decreasing on $\mathbb{R}$ and

$$
\begin{aligned}
\lim _{\alpha \rightarrow+\infty} \mu(\lambda, \alpha) & =-\infty, \\
\lim _{\alpha \rightarrow-\infty} \mu(\lambda, \alpha) & =\lambda_{1}(-\Delta-\lambda m, D) .
\end{aligned}
$$

2. Assume that $m$ changes sign. Then the map $\lambda \mapsto \mu(\lambda, \alpha)$ is concave on $\mathbb{R}$ and $\lim _{|\lambda| \rightarrow \infty} \mu(\lambda, \alpha)=-\infty$. Moreover, it is differentiable and

$$
\frac{d \mu}{d \lambda}(\lambda, \alpha)=-\int_{\Omega} m(x) \phi_{\lambda, \alpha}^{2}
$$

where $\phi_{\lambda, \alpha}$ is the eigenfunction achieving $\mu(\lambda, \alpha)$.

3. For every $\alpha \in \mathbb{R}$ the map $\lambda \mapsto \mu(\lambda, \alpha)$ has an unique maximum point.

We shall first consider $(E)$ as an eigenvalue problem with respect to $\alpha$. It is clear that, given $\lambda \in \mathbb{R}, \alpha_{1}(\lambda)$ is a principal eigenvalue of $(E)$ if and only if $\mu\left(\lambda, \alpha_{1}(\lambda)\right)=0$. From Lemma 2.2 we deduce:

Lemma 2.3. Given $\lambda \in \mathbb{R},(E)$ has a principal eigenvalue $\alpha_{1}(\lambda)$ if and only if $\lambda_{1}(-\Delta-$ $\lambda m, D)>0$. In this case we have

$$
\alpha_{1}(\lambda)=\min \left\{I_{\lambda, 0}(u) ; u \in H^{1}(\Omega), \int_{\partial \Omega} u^{2}=1\right\}
$$

and

$$
\left.\alpha_{1}(\lambda)>0 \quad(\text { respect. }=0,<0) \quad \Longleftrightarrow \quad \lambda_{1}(-\Delta-\lambda m, N)>0 \quad \text { (respect. }=0,<0\right) .
$$

In Figure 5 we have depicted the map $\alpha \mapsto \mu(\lambda, \alpha)$ depending on the values of $\lambda$.

On the other hand, when dealing with $(E)$ as an eigenvalue problem with respect to $\lambda$, we shall consider the maximum of the map $\lambda \mapsto \mu(\lambda, \alpha)$. We complement Lemma 2.2 providing an expression for this maximum, namely:

$$
\mu_{0}(\alpha):=\inf \left\{I_{0, \alpha}(u) ; u \in H^{1}(\Omega), \int_{\Omega} u^{2}=1, \int_{\Omega} m(x) u^{2}=0\right\} .
$$



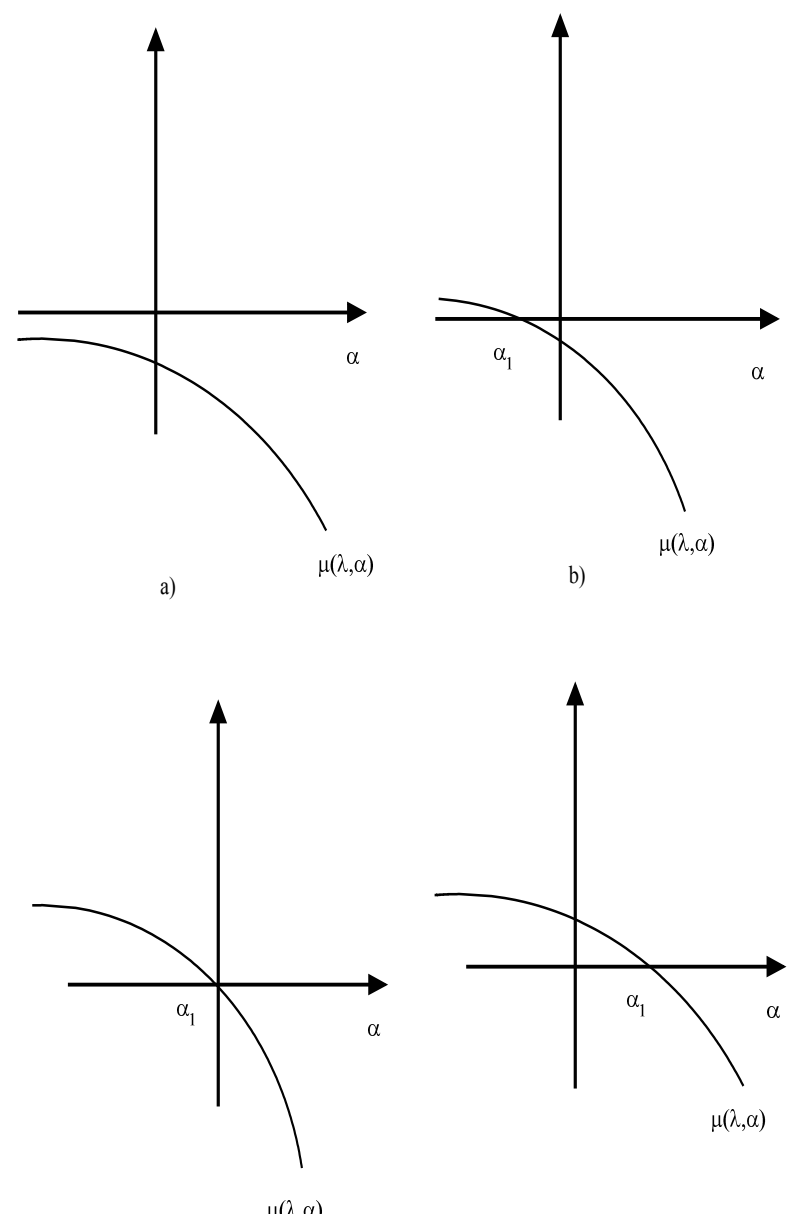

c)

d)

Figure 5: The map $\alpha \mapsto \mu(\lambda, \alpha)$ : Case a) $\lambda_{1}(-\Delta-\lambda m, D) \leq 0$. Case b) $\lambda_{1}(-\Delta-\lambda m, N)<$ $0<\lambda_{1}(-\Delta-\lambda m, D)$. Case c) $\lambda_{1}(-\Delta-\lambda m, N)=0$. Case d) $\lambda_{1}(-\Delta-\lambda m, N)>0$. 
Lemma 2.4. For every $\alpha \in \mathbb{R}$ there holds

$$
\max _{\lambda \in \mathbf{R}} \mu(\lambda, \alpha)=\mu_{0}(\alpha)
$$

Proof. We know that $\lim _{|\lambda| \rightarrow \infty} \mu(\lambda, \alpha)=-\infty$ and $\lambda \mapsto \mu(\lambda, \alpha)$ is continuous, so that it has a global maximum achieved by some $\lambda_{0}$, i.e.

$$
\max _{\lambda \in \mathbf{R}} \mu(\lambda, \alpha)=\mu\left(\lambda_{0}, \alpha\right) .
$$

We shall prove that $\mu\left(\lambda_{0}, \alpha\right)=\mu_{0}(\alpha)$. Since $\lambda \mapsto \mu(\lambda, \alpha)$ is differentiable and

$$
\frac{d \mu}{d \lambda}(\lambda, \alpha)=-\int_{\Omega} m(x) \phi_{\lambda, \alpha}^{2}
$$

where $\phi_{\lambda, \alpha}$ is the eigenfunction achieving $\mu(\lambda, \alpha)$, we must have

$$
\int_{\Omega} m(x) \phi_{0}^{2}=0
$$

where $\phi_{0}=\phi_{\lambda_{0}, \alpha}$. Consequently

$$
\mu\left(\lambda_{0}, \alpha\right)=I_{\lambda_{0}, \alpha}\left(\phi_{0}\right)=I_{0, \alpha}\left(\phi_{0}\right) \geq \mu_{0}(\alpha) .
$$

On the other hand, it is easily seen that $\mu_{0}(\alpha)$ is achieved by some $u_{0}$. Hence

$$
\mu\left(\lambda_{0}, \alpha\right) \leq I_{\lambda_{0}, \alpha}\left(u_{0}\right)=I_{0, \alpha}\left(u_{0}\right)=\mu_{0}(\alpha),
$$

and we get the conclusion.

We are now in position to analyse the existence of zeros for the map $\lambda \mapsto \mu(\lambda, \alpha)$. The case $\alpha=0$ (Neumann) is well-known, whereas the other cases were considered in [1], but we shall provide them a complete and unified description. We set

$$
\alpha_{0}^{*}:=\inf \left\{\int_{\Omega}|\nabla u|^{2} ; u \in H^{1}(\Omega), \int_{\Omega} m(x) u^{2}=0, \int_{\partial \Omega} u^{2}=1\right\} .
$$

Lemma 2.5. Assume (1.1).

1. If $\alpha>\alpha_{0}^{*}$ then $(E)$ has no principal eigenvalues.

2. If $\alpha=\alpha_{0}^{*}$ then $(E)$ has a unique principal eigenvalue $\lambda_{1}(\alpha)$.

3. If $\alpha<\alpha_{0}^{*}$ then $(E)$ has two principal eigenvalues $\lambda_{1}^{-}(\alpha)<\lambda_{1}^{+}(\alpha)$, given by

$$
\lambda_{1}^{ \pm}(\alpha)= \pm \min \left\{I_{0, \alpha}(u) ; u \in H^{1}(\Omega), \int_{\Omega} m(x) u^{2}= \pm 1\right\} .
$$

Moreover:

(a) If $\alpha<0$ then $\lambda_{1}^{-}(\alpha)<0<\lambda_{1}^{+}(\alpha)$.

(b) If $\alpha=0$ then $\lambda_{1}^{-}(\alpha)=0<\lambda_{1}^{+}(\alpha)$.

(c) If $0<\alpha<\alpha_{0}^{*}$ then $0<\lambda_{1}^{-}(\alpha)<\lambda_{1}^{+}(\alpha)$. 
Proof. Since $\lambda$ is a principal eigenvalue of $(E)$ if and only if $\mu(\lambda, \alpha)=0$, we shall look for the zeros of the map $\lambda \mapsto \mu(\lambda, \alpha)$. From Lemma 2.4, we know that $\max _{\lambda \in \mathbf{R}} \mu(\lambda, \alpha)=\mu_{0}(\alpha)$. Thus the condition $\mu_{0}(\alpha) \geq 0$ is necessary for the existence of principal eigenvalues of $(E)$.

Note also from (2.8) that $\mu_{0}(\alpha)>0$ if $\alpha \leq 0$. Now, if $\alpha>0$ then $\mu_{0}(\alpha) \geq 0$ if and only if and only if $I_{0, \alpha}(u)>0$ for every $u \neq 0$ such that $\int_{\Omega} m(x) u^{2}=0$, i.e. if and only if $\alpha \leq \alpha_{0}^{*}$. Moreover, if $\alpha=\alpha_{0}^{*}$ then $\mu_{0}(\alpha)=0$ and, by Lemma 2.4, there is an unique $\lambda_{0}$ such that $\mu\left(\lambda_{0}, \alpha\right)=\mu_{0}(\alpha)$. We set $\lambda_{1}(\alpha)=\lambda_{0}$. On the other hand, if $\alpha<\alpha_{0}^{*}$ then $\lambda \mapsto \mu(\lambda, \alpha)$ vanishes at some $\lambda_{1}^{-}(\alpha)<\lambda_{1}^{+}(\alpha)$. Since

$$
\mu\left(\lambda_{1}^{-}(\alpha), \alpha\right)=0 \quad \text { and } \quad \frac{d \mu}{d \lambda}\left(\lambda_{1}^{-}(\alpha), \alpha\right)>0,
$$

we have, denoting $\phi=\phi_{\lambda_{1}^{-}(\alpha), \alpha}$,

$$
I_{\lambda_{1}^{-}(\alpha), \alpha}(u) \geq 0 \text { for every } u \in H^{1}(\Omega), \quad I_{\lambda_{1}^{-}(\alpha), \alpha}(\phi)=0 \quad \text { and } \quad \int_{\Omega} m(x) \phi^{2}<0 .
$$

Let $\psi=\left(-\int_{\Omega} m(x) \phi^{2}\right)^{-\frac{1}{2}} \phi$. Then $\int_{\Omega} m(x) \psi^{2}=-1$ and, from $I_{\lambda_{1}^{-}(\alpha), \alpha}(\phi)=0$, we get

$$
-\lambda_{1}^{-}(\alpha)=I_{0, \alpha}(\psi) \text {. }
$$

Moreover, since $I_{\lambda_{1}^{-}(\alpha), \alpha}(u) \geq 0$ for every $u \in H^{1}(\Omega)$, we have in particular

$$
-\lambda_{1}^{-}(\alpha) \leq I_{0, \alpha}(u) \quad \text { for every } u \text { such that } \int_{\Omega} m(x) u^{2}=-1
$$

Thus

$$
-\lambda_{1}^{-}(\alpha)=\min \left\{I_{0, \alpha}(u) ; u \in H^{1}(\Omega), \int_{\Omega} m(x) u^{2}=-1\right\} .
$$

In a similar way, we can prove that

$$
\lambda_{1}^{+}(\alpha)=\min \left\{I_{0, \alpha}(u) ; u \in H^{1}(\Omega), \int_{\Omega} m(x) u^{2}=1\right\} .
$$

Finally, note from (2.11) that the map $\alpha \mapsto-\lambda_{1}^{-}(\alpha)$ is decreasing on $\left(-\infty, \alpha_{0}^{*}\right)$ and $\lambda_{1}^{-}(0)=0$, in view of (1.1). Therefore $\lambda_{1}^{-}(\alpha)>0$ if and only if $0<\alpha<\alpha_{0}^{*}$. In a similar way, $\alpha \mapsto \lambda_{1}^{+}(\alpha)$ is decreasing on $\left(-\infty, \alpha_{0}^{*}\right)$ and $\lambda_{1}^{+}(\alpha)>\lambda_{1}^{-}(\alpha)>0$ if $0<\alpha<\alpha_{0}^{*}$, so that $\lambda_{1}^{+}(\alpha)>0$ for every $\alpha<\alpha_{0}^{*}$.

In the following result, we compare the maps $\alpha_{1}(\lambda)$ and $\lambda_{1}^{ \pm}(\alpha)$. It can be easily proved using Lemmas 2.2 and 2.3, and the fact that, whenever $\alpha_{1}(\lambda)$ and $\lambda_{1}^{ \pm}(\alpha)$ exist, we have

$$
\alpha<\alpha_{1}(\lambda) \Leftrightarrow \mu(\lambda, \alpha)>0 \Leftrightarrow \lambda_{1}^{-}(\alpha)<\lambda<\lambda_{1}^{+}(\alpha) .
$$

Lemma 2.6. Assume (1.1) and $\alpha<\alpha_{0}^{*}$. Then

1. $\alpha<\alpha_{1}(\lambda) \Leftrightarrow \lambda \in\left(\lambda_{1}^{-}(\alpha), \lambda_{1}^{+}(\alpha)\right)$.

2. If $\alpha>\alpha_{1}(\lambda)$ and $\lambda<0$, then $\lambda<\lambda_{1}^{-}(\alpha)$.

3. If $\alpha>\alpha_{1}(\lambda), \lambda>0$ and:

(a) $\alpha \leq 0$, then $\lambda>\lambda_{1}^{+}(\alpha)$.

(b) $\alpha>0$, then either $0<\lambda<\lambda_{1}^{-}(\alpha)$ or $\lambda>\lambda_{1}^{+}(\alpha)$. 


\section{Bifurcation with respect to $\alpha$}

Let us recall that a positive solution $u_{0}$ of $(P)$ is stable if the principal eigenvalue of the linearisation of $(P)$ at $u_{0}$ is positive, i.e.

$$
\lambda_{1}\left(-\Delta-\lambda m+2 u_{0} \lambda m, N-\alpha\right)>0 .
$$

Since $\alpha_{1}(\lambda)$ is a simple eigenvalue whenever it exists, i.e., if $\lambda \in\left(\lambda_{1}^{-}(D), \lambda_{1}^{+}(D)\right)$, we can apply the classical Crandall-Rabinowitz Theorem [9] to deduce the following result:

Lemma 3.1. Assume that there exists $\alpha_{1}(\lambda)$. Then:

1. The trivial solution $u \equiv 0$ is stable for $\alpha<\alpha_{1}(\lambda)$ and unstable for $\alpha>\alpha_{1}(\lambda)$.

2. The point $(\alpha, u)=\left(\alpha_{1}(\lambda), 0\right)$ is a bifurcation point from the trivial solution of $(P)$. Moreover, there exist $\varepsilon>0$ and two $C^{1}$ maps

$$
\alpha:(-\varepsilon, \varepsilon) \mapsto \mathbb{R} \quad \text { and } \quad v:(-\varepsilon, \varepsilon) \mapsto\left\langle\varphi_{1}\right\rangle^{\perp},
$$

where $\varphi_{1}$ is a positive eigenfunction associated to $\alpha_{1}(\lambda)$, satisfying $\alpha(0)=\alpha_{1}(\lambda)$, $v(0)=0$ and

$$
\alpha(s)=\alpha_{1}(\lambda)+s \alpha_{2}+o(s), \quad u(s)=s\left(\varphi_{1}+v(s)\right)
$$

are such that $(\alpha(s), u(s))$ is the only solution of $(P)$ in a neighborhood of $\left(\alpha_{1}(\lambda), 0\right)$. Moreover,

$$
\alpha_{2}=\frac{\lambda \int_{\Omega} m \varphi_{1}^{3}}{\int_{\Omega} \varphi_{1}^{2}} .
$$

Consequently, for $\lambda \neq 0$, the bifurcation direction is supercritical (resp. subcritical) if $\alpha_{2}>0\left(\right.$ resp. $\left.\alpha_{2}<0\right)$.

3. If the bifurcation direction is supercritical (respect. subcritical) the new solution $u(s)$ is stable (respect. unstable).

4. There exists $\delta>0$ such that $\alpha_{2}>0$ if $\lambda \in\left(\lambda_{1}^{-}(D)-\delta, 0\right) \cup\left(\lambda_{1}^{+}(0)-\delta, \lambda_{1}^{+}(D)+\delta\right)$. In particular $\alpha_{2}>0$ if $\alpha_{1}(\lambda)=0$.

Proof. Observe that $u \equiv 0$ is stable if $\lambda_{1}(-\Delta-\lambda m, N-\alpha)>0$, that is $\alpha<\alpha_{1}(\lambda)$. The existence and properties of the maps $\alpha(s)$ and $u(s)$ follow by the Crandall-Rabinowitz Theorem. In addition, since $(\alpha(s), u(s))$ solve $(P)$, we have

$$
\begin{cases}-\Delta\left(\left(\varphi_{1}+v(s)\right)\right)=\lambda m(x)\left(\varphi_{1}+v(s)\right)\left(1-s\left(\varphi_{1}+v(s)\right)\right) & \text { in } \Omega, \\ \frac{\partial\left(\varphi_{1}+v(s)\right)}{\partial \nu}=\left(\alpha_{1}(\lambda)+s \alpha_{2}+o(s)\right)\left(\varphi_{1}+v(s)\right) & \text { on } \partial \Omega .\end{cases}
$$

We can write $v(s)=s v_{1}+s^{2} v_{2}+o\left(s^{2}\right)$ for $s \simeq 0$. Plugging this expression in the above equation and rearranging the terms in $s$, we get

$$
-\Delta v_{1}-\lambda m(x) v_{1}=-\lambda m(x) \varphi_{1}^{2} \quad \text { in } \Omega, \quad \frac{\partial v_{1}}{\partial \nu}-\alpha_{1}(\lambda) v_{1}=\alpha_{2} \varphi_{1} \quad \text { on } \partial \Omega
$$


and multiplying by $\varphi_{1}$, we get

$$
\alpha_{2}=\frac{\lambda \int_{\Omega} m \varphi_{1}^{3}}{\int_{\Omega} \varphi_{1}^{2}} .
$$

Now, since

$$
-\Delta \varphi_{1}-\lambda m(x) \varphi_{1}=0 \quad \text { in } \Omega, \quad \frac{\partial \varphi_{1}}{\partial \nu}=\alpha_{1}(\lambda) \varphi_{1} \quad \text { on } \partial \Omega,
$$

multiplying by $\varphi_{1}^{2}$, we get

$$
2 \int_{\Omega} \varphi_{1}\left|\nabla \varphi_{1}\right|^{2}-\alpha_{1}(\lambda) \int_{\partial \Omega} \varphi_{1}^{3}=\lambda \int_{\Omega} m \varphi_{1}^{3}
$$

Recall that $\alpha_{1}(\lambda)<0$ for $\lambda \in\left(\lambda_{1}^{-}(D), 0=\lambda_{1}^{-}(0)\right) \cup\left(\lambda_{1}^{+}(0), \lambda_{1}^{+}(D)\right)$. Thus, by continuity of $\alpha_{1}(\lambda)$ and $\varphi_{1}$ with respect to $\lambda$ (see for instance [8]), there exists $\delta>0$ such that $\alpha_{2}>0$ for $\lambda \in\left(\lambda_{1}^{-}(D)-\delta, 0\right) \cup\left(\lambda_{1}^{+}(0)-\delta, \lambda_{1}^{+}(D)+\delta\right)$. In particular, $\alpha_{2}>0$ when $\alpha_{1}(\lambda)=0$, that is, when $\lambda=\lambda_{1}^{+}(0)$.

Finally, the stability of the new solution $u(s)$ follows by [10].

The following result has a global character (see [15]):

Lemma 3.2. Whenever $\alpha_{1}(\lambda)$ exists, there is an unbounded continuum $\mathcal{C}$ of positive solutions of $(P)$ emanating from $(\alpha, u)=\left(\alpha_{1}(\lambda), 0\right)$.

In the following result, we prove that $(P)$ has no positive solutions for $\alpha$ large and independent of $\lambda$. Let $\alpha_{0}$ be the principal eigenvalue of

$$
\begin{cases}-\Delta u=0 & \text { in } M_{0} \\ \frac{\partial u}{\partial \nu}=\alpha u & \text { on } \partial M_{0} \cap \partial \Omega \\ u=0 & \text { on } \partial M_{0} \backslash \partial \Omega\end{cases}
$$

where $M_{0}$ is given in (1.2).

Remark 3.3. Note that (3.12) has indeed a principal eigenvalue $\alpha_{0}$. This can be proved in the same way as the existence of $\alpha_{1}(\lambda)$ in Lemma 2.3. As a matter of fact, if we denote by $\mu_{1}(\alpha)$ the principal eigenvalue of the problem

$$
\begin{cases}-\Delta u=\mu_{1}(\alpha) u & \text { in } M_{0} \\ \frac{\partial u}{\partial \nu}-\alpha u=0 & \text { on } \partial M_{0} \cap \partial \Omega \\ u=0 & \text { on } \partial M_{0} \backslash \partial \Omega\end{cases}
$$

then $\alpha_{0}$ is a principal eigenvalue of (3.12) if and only if $\mu_{1}\left(\alpha_{0}\right)=0$. Now, since $\alpha \mapsto \mu_{1}(\alpha)$ is decreasing, $\mu_{1}(0)>0$ and $\lim _{\alpha \rightarrow \infty} \mu_{1}(\alpha)=-\infty$, we deduce the existence and uniqueness of $\alpha_{0}$. 
Lemma 3.4. If $\alpha \geq \alpha_{0}$ then $(P)$ has no positive solution.

Proof. Let $u$ be a positive solution of $(P)$. Since $M_{0}$ is a proper subdomain of $\Omega$, by Lemma 2.1 we have

$$
0=\lambda_{1}(-\Delta-\lambda m+\lambda m u, N-\alpha) \leq \lambda_{1}^{M_{0}}(-\Delta, N-\alpha, D),
$$

which implies $\alpha<\alpha_{0}$.

We shall take advantage of the results known for $(P)$ when $\alpha=0$, as shown in [6]:

Lemma 3.5. Assume (1.1) and $\alpha=0$. Then:

1. $(P)$ has two trivial solutions, $u \equiv 0$ and $u \equiv 1$, for all $\lambda \in \mathbb{R}$. Moreover, $u \equiv 0$ is stable for $\lambda \in\left(0, \lambda_{1}^{+}(0)\right)$ and $u \equiv 1$ is stable for $\lambda \in\left(-\lambda_{1}^{+}(0), 0\right)$.

2. $(P)$ has a stable positive solution $u_{\lambda}$ for $\lambda \in\left(-\infty,-\lambda_{1}^{+}(0)\right) \cup\left(\lambda_{1}^{+}(0),+\infty\right)$. Moreover, $u_{\lambda}<1$ and this is the only positive solution of $(P)$ satisfying $u<1$.

Proof.

1. It is clear that $u=0$ and $u=1$ solve $(P)$. The stability of $u=0$ follows by Theorem 3 in [6], whereas the linearized problem around $u=1$ is

$$
-\Delta w=(-\lambda) m(x) w \quad \text { in } \Omega, \quad \frac{\partial w}{\partial \nu}=0 \quad \text { on } \partial \Omega,
$$

for which the first eigenvalue is positive if and only $-\lambda \in\left(0, \lambda_{1}^{+}(0)\right)$.

2. The case $\lambda \in\left(\lambda_{1}^{+}(0),+\infty\right)$ follows by [6]. On the other hand, observe that $w=1-u$ verifies

$$
-\Delta w=(-\lambda) m(x) w(1-w) \quad \text { in } \Omega, \quad \frac{\partial w}{\partial \nu}=0 \quad \text { on } \partial \Omega,
$$

so that, by [6], there exists a unique stable solution $0<w_{\lambda}<1$ for $-\lambda \in\left(\lambda_{1}^{+}(0),+\infty\right)$, that is, for $\lambda \in\left(-\infty,-\lambda_{1}^{+}(0)\right)$.

Let us set $\mathcal{E}:=\mathcal{C}(\bar{\Omega}), \mathcal{P}:=\{u \in \mathcal{E} ; u \geq 0\}$ and

$$
\Sigma=:\{(\alpha, u) \in \mathbb{R} \times \mathcal{P} ; u \text { is a positive solution of }(P)\} .
$$

Lemma 3.6. Assume that $u_{0}$ is a positive stable solution of $(P)$ for $\alpha=0$. Then:

1. There exist $\varepsilon>0$ and a neighborhood $\mathcal{U} \subset \mathbb{R} \times \mathcal{P}$ such that $\mathcal{U} \cap \Sigma=\left\{\left(\alpha, u_{\alpha}\right) ; \alpha \in\right.$ $(-\varepsilon, \varepsilon)\}$. Morever, $u_{\alpha}$ is stable for $\alpha \in(-\varepsilon, \varepsilon)$.

2. There exists an unbounded continuum $\mathcal{C}_{0}$ of positive solutions of $(P)$ containing $\left(0, u_{0}\right)$. Moreover, if we assume that there exist a priori bounds for positive solutions of $(P)$ whenever $\alpha$ varies in a compact set, then there exists $\mathcal{F} \subset \mathcal{P}$ such that

$$
\mathcal{C}_{0} \cap(\{0\} \times \mathcal{F}) \neq \emptyset, \quad \mathcal{C}_{0} \cap(\{0\} \times(\mathcal{P} \backslash \mathcal{F})) \neq \emptyset .
$$

Proof. Since $u_{0}$ is stable, the first result follows by Proposition 20.6 in [3] and the existence of $\mathcal{C}_{0}$ containing $\left(0, u_{0}\right)$ by Theorem 17.1 in [3]. The second paragraph is a consequence of the first one and Lemma 3.4. See also [5], Theorems 4.4.1 and 4.4.2 for the first and second paragraphs, respectively. 


\subsection{A priori bounds}

In this section we get a priori bounds for positive solutions of $(P)$ when $\alpha$ belongs to a compact set of $\mathbb{R}$.

Proposition 3.7. Let $\lambda>0$. Assume that there exist a function $h^{-}: \overline{M_{-}} \mapsto \mathbb{R}^{+}$, continuous and bounded away from zero in a neighborhood of $\partial M_{-}$, and a constant $\gamma_{-} \geq 0$ such that

$$
m^{-}(x)=h^{-}(x)\left(\operatorname{dist}\left(x, \partial M_{-}\right)\right)^{\gamma_{-}} \quad \text { in } M_{-} .
$$

Assume in addition

$$
2<\min \left\{\frac{N+1+\gamma_{-}}{N-1}, \frac{N+2}{N-2}\right\} \quad \text { if } N \geq 3 .
$$

Then, for every compact interval $\Lambda \subset \mathbb{R}$ there exists a positive constant $C$ such that

$$
\|u\|_{\infty} \leq C
$$

for any positive solution $u$ of $(P)$ with $\alpha \in \Lambda$.

Proof. First note that if $(P)$ has positive solution $u$ then, by Lemma 3.4, we must have $\alpha<\alpha_{0}$.

We split the proof in two steps.

Step 1: A priori bounds on $\overline{M_{-}}$. For this step, we use (3.14), an adequate rescaling Gidas-Spruck argument and a Liouville type theorem, see exactly Lemma 4.2 and Theorem 4.3 of [4].

Step 2: A priori bounds on $\Omega$. Define

$$
R:=\sup _{\alpha \in \Lambda} \sup _{x \in \bar{M}_{-}} u(x)<\infty .
$$

We consider the problem

$$
\begin{cases}-\Delta u=\lambda m(x) u(1-u) & \text { in } \Omega \backslash \overline{M_{-}} \\ u=R & \text { on } \partial\left(M_{-}\right) \backslash \partial \Omega, \\ \frac{\partial u}{\partial \nu}=\alpha u & \text { on } \partial\left(\Omega \backslash \bar{M}_{-}\right) \cap \partial \Omega .\end{cases}
$$

We claim that there exists a unique positive solution $U$ of (3.15) for all $\alpha<\alpha_{0}$. In this case it is clear that a solution $u$ of $(P)$ is a subsolution of (3.15) in $\Omega \backslash \overline{M_{-}}$. By the uniqueness of the positive solution of $(3.15)$ we get

$$
\|u\|_{L^{\infty}\left(\Omega \backslash \bar{M}_{-}\right)} \leq\|U\|_{L^{\infty}\left(\Omega \backslash \bar{M}_{-}\right)},
$$

whence the result follows.

It remains to prove the claim. We use the sub-supersolution method to obtain $U$. Indeed, $\underline{u}:=0$ is a subsolution of (3.15). Now, set

$$
M_{\delta}:=\left\{x \in \Omega: \operatorname{dist}\left(x, M_{0}\right)<\delta\right\},
$$


for $\delta>0$, and consider the eigenvalue problem

$$
\begin{cases}-\Delta u=0 & \text { in } M_{\delta} \\ u=0 & \text { on } \partial M_{\delta} \cap \Omega, \\ \frac{\partial u}{\partial \nu}=\alpha u & \text { on } \partial \Omega .\end{cases}
$$

Thanks to Remark 3.3 there exists a principal eigenvalue $\alpha_{1}(\delta)$ of $(3.16)$, and $\varphi_{\delta}$ a positive eigenfunction associated to $\alpha_{1}(\delta)$. Now, we can show that $M_{\delta}$ is a sequence of bounded and regular domains converging to $M_{0}$ from the exterior in the sense of [8]. So, by Theorem 7.1 in [8], we conclude that

$$
\alpha_{1}(\delta) \uparrow \alpha_{0} \quad \text { as } \delta \uparrow 0 .
$$

Take $\alpha<\alpha_{0}$ and consider $\delta$ such that $\alpha<\alpha_{1}(\delta)<\alpha_{0}$. Now, define

$$
\Psi:= \begin{cases}\varphi_{\delta} & \text { in } M_{\delta / 2} \cap\left(\Omega \backslash \bar{M}_{-}\right), \\ \psi & \text { in } M_{+} \backslash M_{\delta / 2},\end{cases}
$$

where $\psi$ is a smooth and positive function such that $\Psi$ is smooth. Then, $\bar{u}:=K \Psi$ is a supersolution of (3.15) for $K$ sufficiently large. Indeed, it is clear that $K \Psi$ is supersolution in $M_{0}$ because $-\Delta(K \Psi)=0$ in $M_{0}$. In $M_{+} \cap M_{\delta / 2}$ we have

$$
-\Delta(K \Psi)=0 \geq \lambda m(x) K \varphi_{\delta}\left(1-K \varphi_{\delta}\right) \quad \text { for } K \text { large. }
$$

Moreover, in $M_{+} \backslash M_{\delta / 2}$ we get

$$
-\Delta(K \Psi)=K(-\Delta(\psi)) \geq \lambda m(x) K \psi(1-K \psi) \text { for } K \text { large. }
$$

On $\partial M_{-}$, we take $K$ such that $K \Psi \geq R$. Thus, it is clear that

$$
\frac{\partial \bar{u}}{\partial \nu}=\frac{\partial(K \Psi)}{\partial \nu}=K \frac{\partial \varphi_{d}}{\partial \nu}=K \alpha_{1} \varphi_{\delta}>\alpha \bar{u} \quad \text { on } \partial \Omega
$$

Finally, the uniqueness follows by Theorem 1.2 in [16].

By symmetry on $\lambda$, we deduce the following a priori bounds result for positive solutions of $(P)$.

Theorem 3.8. Let $\lambda \neq 0$. Assume that there exist two functions $h^{ \pm}: \overline{M_{ \pm}} \mapsto \mathbb{R}^{+}$, continuous and bounded away from zero in a neighborhood of $\partial M_{ \pm}$, and constants $\gamma_{ \pm} \geq 0$ such that

$$
m^{ \pm}(x)=h^{ \pm}(x)\left(\operatorname{dist}\left(x, \partial M_{ \pm}\right)\right)^{\gamma_{ \pm}} \quad \text { in } M_{ \pm} .
$$

Assume in addition

$$
2<\min \left\{\frac{N+1+\gamma_{ \pm}}{N-1}, \frac{N+2}{N-2}\right\} \quad \text { if } N \geq 3 .
$$

Then, for every compact interval $\Lambda \subset \mathbb{R}$ there exists a positive constant $C$ such that

$$
\|u\|_{\infty} \leq C
$$

for any positive solution $u$ of $(P)$ with $\alpha \in \Lambda$. 


\section{Proof of the main results}

Before proving our main results, we need the following result (recall the definition of $\mu(\lambda, \alpha)$ in $(2.5))$.

\section{Lemma 4.1.}

1. Assume that there exists a positive solution $u_{*}$ of $(P)$ for $\alpha=\alpha_{*}$. Then, there exists a positive solution for every $\alpha<\alpha_{*}$ such that $\mu(\lambda, \alpha)<0$.

2. Assume that there exists a positive solution $u_{0}$ of $(P)$ for $\alpha=0$. Then, there exists a positive solution $u_{\alpha}$ for all $\alpha<0$ such that $\mu(\lambda, \alpha)<0$. Moreover, if $u_{0} \leq 1$ then $u_{\alpha}$ is stable.

Proof.

1. We use the sub-supersolution method. Consider $\varphi$ a positive eigenfunction associated to $\mu(\lambda, \alpha)$. Take as pair of sub-supersolution $(\underline{u}, \bar{u})=\left(\varepsilon \varphi, u_{*}\right)$, with $\varepsilon>0$. It is easily seen that $\underline{u}$ is a sub-solution of $(P)$ if

$$
\mu(\lambda, \alpha)+\lambda m(x) \varepsilon \varphi \leq 0,
$$

which holds for $\varepsilon$ small enough. Then, there exists a positive solution $u_{\alpha} \in\left(\underline{u}, u_{*}\right)$.

2. The existence of $u_{\alpha}$ follows by the previous item. Assume that $u_{0} \leq 1$. Since $u \equiv 1$ is not solution of $(P)$ for $\alpha<0$, we have $u_{\alpha}<1$. Now, we show that $u_{\alpha}$ is stable, i.e.

$$
\lambda_{1}\left(-\Delta-\lambda m(x)\left(1-2 u_{\alpha}\right), N-\alpha\right)>0 .
$$

To this end, we prove the existence of a positive supersolution for the operator $\left(-\Delta-\lambda m(x)\left(1-2 u_{\alpha}\right), N-\alpha\right)$. Take $\bar{u}:=f\left(u_{\alpha}\right)$ where $f\left(u_{\alpha}\right)=u_{\alpha}\left(1-u_{\alpha}\right)>0$, see [6]. Then, it is clear that

$$
-\Delta \bar{u}-\lambda m(x)\left(1-2 u_{\alpha}\right) \bar{u}=-f^{\prime \prime}\left(u_{\alpha}\right)\left|\nabla u_{\alpha}\right|^{2}>0 \quad \text { in } \Omega,
$$

and

$$
\frac{\partial \bar{u}}{\partial \nu}-\alpha \bar{u}=-2 \alpha u_{\alpha}^{2}>0 \quad \text { on } \partial \Omega .
$$

This proves that $u_{\alpha}$ is stable.

We are now ready to prove Theorem 1.1:

\section{Proof of Theorem 1.1 .}

1. Assume that $\lambda_{1}(-\Delta-\lambda m, D)>0$. By Lemma 2.3 we know that $(E)$ has a principal eigenvalue $\alpha_{1}(\lambda)$ and from Lemma 3.2 there exists an unbounded continuum $\mathcal{C}$ of positive solutions of $(P)$ emanating from $(\alpha, u)=\left(\alpha_{1}(\lambda), 0\right)$. On the other hand, by Lemma 3.4 there is no positive solution of $(P)$ for $\alpha \geq \alpha_{0}$. Moreover, Theorem 3.8 provides us with a priori bounds for positive solutions of $(P)$, so we conclude the existence of positive solutions of $(P)$ for all $\alpha<\alpha_{1}$. 
Now, we set

$$
\alpha_{*}:=\sup \{\alpha \in \mathbb{R}:(P) \text { has a positive solution }\}
$$

It is clear that $\alpha_{*}<\infty$. Thanks to the a priori bounds, we infer the existence of a non-negative solution of $(P)$ for $\alpha=\alpha_{*}$, which we denote by $u_{*}$.

If $\alpha_{*}=\alpha_{1}(\lambda)$ we conclude the existence of positive solution for $\alpha<\alpha_{*}$ and no positive solution for $\alpha>\alpha_{*}$.

Assume that $\alpha_{*}>\alpha_{1}(\lambda)$. In this case, since $\alpha_{1}(\lambda)$ is the unique bifurcation point from the trivial solution, we can show that $u_{*}>0$. Now, by Lemma 4.1, we know that $(P)$ has a positive solution $u_{\alpha}$ for every $\alpha<\alpha_{*}$ such that $\mu(\lambda, \alpha)<0$, that is, for $\alpha \in\left(\alpha_{1}, \alpha_{*}\right)$.

On the other hand, by Lemma 3.1 the solution $u_{\alpha}$ is stable for $\alpha \in\left(\alpha_{1}(\lambda), \alpha_{1}(\lambda)+\delta\right)$ for some $\delta>0$. This implies the existence of two positive solutions of $(P)$ for $\alpha \in\left(\alpha_{1}(\lambda), \alpha_{1}(\lambda)+\delta\right)$ and the existence of positive solution for all $\alpha \leq \alpha_{*}$.

(a) Assume that $\lambda_{1}(-\Delta-\lambda m, N)>0$. Then, in this case $\alpha_{1}(\lambda)>0$ and so $\alpha_{*}>0$.

(b) If $\lambda_{1}(-\Delta-\lambda m, N)=0$ then $\alpha_{1}(\lambda)=0$. In this case, by Lemma 3.1, the direction of bifurcation is supercritical, and so again $\alpha_{*}>0=\alpha_{1}(\lambda)$.

(c) Assume now that $\lambda_{1}(-\Delta-\lambda m, N)<0$, that is, $\lambda>\lambda_{1}^{+}(0)$ or $\lambda<\lambda_{1}^{-}(0)=0$ and $\lambda \neq-\lambda_{1}^{+}(0)$. Recall that in this case $\alpha_{1}<0$. Hence, by Lemma 3.5, for $\alpha=0$ there exists a stable solution $u_{0} \leq 1$ of $(P)$. By Lemma 4.1, we have a stable positive solution $u_{\alpha}$ for all $\alpha \in\left(\alpha_{1}, 0\right]$. By continuity, we have a stable solution, still denoted $u_{\alpha}$, for $\alpha \in\left(\alpha_{1}, \alpha_{* *}\right)$. Now, in view of non-existence of solutions for large $\alpha$, the continuum $\mathcal{C}_{0}$ has to turn backwards, and so we conclude the existence of a second solution, $w_{\alpha}$, for $\alpha \in\left(\alpha_{1}, \alpha_{* *}\right)$.

(d) Finally, assume that $\lambda=-\lambda_{1}^{+}(0)$. Again we have $\alpha_{1}(\lambda)<0$ and by Lemma 3.5 for $\alpha=0$ there exists the trivial solution $u_{0} \equiv 1$ of $(P)$. By Lemma 4.1, we have a stable positive solution $u_{\alpha}$ for all $\alpha \in\left(\alpha_{1}(\lambda), 0\right]$. Hence, in this case, $\alpha_{* *} \geq 0$.

2. Assume now that $\lambda_{1}(-\Delta-\lambda m, D) \leq 0$, which implies that $\lambda_{1}(-\Delta-\lambda m, N)<0$. In this case, $\alpha_{1}(\lambda)$ does not exist. However, by Lemma 3.5, for $\alpha=0$ there exists a stable solution $u_{0} \leq 1$ of $(P)$, and consequently, by Lemma 3.6, there exists an unbounded continuum $\mathcal{C}_{0}$ containing $\left(0, u_{0}\right)$ and at least a positive solution for $\alpha \in(-\varepsilon, \varepsilon)$. We set $\alpha_{*}$ as in the previous case. Now, by Lemma 4.1, there exists a positive solution for every $\alpha<\alpha_{*}$ such that $\mu(\lambda, \alpha)<0$, that is, for all $\alpha<\alpha_{*}$. Indeed, since $\lambda_{1}(-\Delta-\lambda m, D) \leq 0$, we have $\mu(\lambda, \alpha)<0$ for all $\alpha$. Moreover, there exists a stable solution for all $\alpha \in\left(-\infty, \alpha_{* *}\right)$. This implies the existence of a second solution in this interval.

Proof of Theorem 1.2.

Items 1 and 2 follow by Lemma 3.5. Moreover, for $\lambda \in\left(-\lambda_{1}^{+}(0), 0\right)$ there holds

$$
\lambda_{1}(-\Delta-\lambda m, N)<0<\lambda_{1}(-\Delta-\lambda m, D)
$$

so that, by Theorem 1.1, there exist two positive solutions for $\alpha=0$. 
Proof of Theorem 1.3.

1. This item follows directly from Lemma 3.4.

2. Assume that $\lambda<0, \lambda \neq-\lambda_{1}^{+}(0)$. In this case, $\lambda_{1}(-\Delta-\lambda m, N)<0$ and applying Theorem 1.1 (in both cases $\lambda_{1}(-\Delta-\lambda m, D) \leq 0$ and $\lambda_{1}(-\Delta-\lambda m, D)>0$ ) we conclude that there exist two positive solutions of $(P)$ for $\alpha$ small enough.

3. Assume now $\lambda \in\left(\lambda_{1}^{-}(\alpha), \lambda_{1}^{+}(\alpha)\right)$. Then $\lambda_{1}(-\Delta-\lambda m, N)>0$, so $\alpha_{1}(\lambda)>0$ and there exists at least a positive solution for $\alpha<\alpha_{1}(\lambda)$, that is, for $\lambda \in\left(\lambda_{1}^{-}(\alpha), \lambda_{1}^{+}(\alpha)\right)$, by Lemma 2.6 .

4. Assume that $\lambda>\lambda_{1}^{+}(0)$. In this case $\lambda_{1}(-\Delta-\lambda m, N)<0$ and again by Theorem 1.1 we conclude that $(P)$ has at least two positive solutions for $\alpha$ small enough. Finally, for $\lambda=\lambda_{1}^{+}(0)$ we have $\alpha_{1}(\lambda)=0$, so that for $\alpha$ sufficiently small, $(P)$ has at least two positive solutions.

Acknowledgements. Supported by Spanish Government under the project MTM201231304 (AS) and Fondecyt 11121567.

\section{References}

[1] G. A. Afrouzi and K. J. Brown, On principal eigenvalues for boundary value problems with indefinite weight and Robin boundary conditions, Proc. Amer. Math. Soc., 127 (1999), 125-130.

[2] G. A. Afrouzi and K. J. Brown, Positive mountain pass solutions for a semilinear elliptic equation with a sign-changing weight function, Nonlinear Anal., 64 (2006), 409-416.

[3] H. Amann, Fixed points equations and nonlinear eigenvalue problems in ordered Banach spaces, SIAM Review, 18 (1976), 620-709.

[4] H. Amann and J. López-Gómez, A priori bounds and multiple solutions for superlinear indefinite elliptic problems, J. Differential Equations, 146 (1998), 336-374.

[5] A. Ambrosetti and D. Arcoya, An introduction to nonlinear functional analysis and elliptic problems. Progress in Nonlinear Differential Equations and their Applications, 82. Birkhuser Boston, Inc., Boston, MA, 2011.

[6] K. J. Brown and P. Hess, Stability and uniqueness of positive solutions for a semilinear elliptic boundary value problem, Differential Integral Equations, 3 (1990), 201207.

[7] K. J. Brown and B. Ko, The existence of positive solutions for a class of indefinite weight semilinear elliptic boundary value problem, Nonlinear Anal., 39 (2000), 587597. 
[8] S. Cano-Casanova and J. López-Gómez, Properties of the principal eigenvalues of a general class of non-classical mixed boundary value problems, J. Differential Equations, 178 (2002), 123-211.

[9] M. G. Crandall and P. H. Rabinowitz, Bifurcation from simple eigenvalues, J. Functional Analysis, 8 (1971) 321-340.

[10] M. G. Crandall and P. H. Rabinowitz, Bifurcation, perturbation of simple eigenvalues and linearized stability, Arch. Rational Mech. Anal., 52 (1973), 161-180.

[11] M. Delgado and A. Suárez, On the existence and multiplicity of positive solutions for some indefinite nonlinear eigenvalue problem. Proc. Amer. Math. Soc., 132 (2004), $1721-1728$

[12] J. García-Melián, J. D. Rossi and J. Sabina de Lis, A bifurcation problem governed by the boundary condition I, NoDEA Nonlinear Differential Equations Appl., 14 (2007), 499-525.

[13] B. Gidas and J. Spruck, A priori bounds for positive solutions of nonlinear elliptic equations, Comm. Partial Differential Equations, 6 (1981), 883-901.

[14] Y. Lou, T. Nagylak and W-M. Ni, An introduction to migration-selection PDE models, Discrete Contin. Dyn. Syst., 33 (2013), 4349-4373.

[15] C. Morales-Rodrigo and A. Suárez, Some elliptic problems with nonlinear boundary conditions, in "Spectral Theory and Nonlinear Analysis with Applications to Spatial Ecology" (S. Cano-Casanova, J. López-Gómez, C. Mora-Corral, eds), World Scientific, (2005), 175-199.

[16] C. Morales-Rodrigo and A. Suárez, Uniqueness of solution for elliptic problems with nonlinear boundary conditions, Comm. Appl. Nonlinear Anal., 13 (2006), 69-78.

[17] S. Senn, On a nonlinear elliptic eigenvalue problem with Neumann boundary conditions, with an application to population genetics, Comm. Partial Differential Equations, 8 (1983), 1199-1228. 